\title{
Microtubule Motional Diffusion Coefficient in Motility Assays is Determined by Heterogeneity in Motor Stiffness
}

\author{
Henri Palacci ${ }^{1, \#}$, Ofer Idan ${ }^{1, \#}$, Megan J. Armstrong ${ }^{1}$, Takahiro Nitta ${ }^{2}$, and Henry Hess ${ }^{1}$ \\ ${ }^{1}$ Department of Biomedical Engineering, Columbia University, New York, NY 10027 \\ ${ }^{2}$ Applied Physics Course, Gifu University, Gifu 501-1193, Japan
}

hp2393@columbia.edu

\begin{abstract}
ABSTRA CT
In the past twenty years, our ability to manipulate and engineer devices at the nano-scale has grown exponentially. As the fabrication of autonomous systems at these scales becomes a reality, the observation of biological structures can help us understand general design principles at the nano-scale. The gliding motility assay is an excellent model system for the observation of collective behavior of coupled motors. Indeed, hundreds of surface-adhered kinesin motors propel one microtubule filament (Figure 1). Filament motion has been observed using fluorescence microscopy, revealing fluctuations in gliding velocity $[3 ; 4]$. We here theoretically characterize the motional diffusion coefficients through the heterogeneity factor proposed by Sekimoto and Tawada [5], and use a Brownian dynamics simulation of kinesin head diffusion under an anharmonic potential to determine a theoretical value of 0.3 for this heterogeneity factor.
\end{abstract}

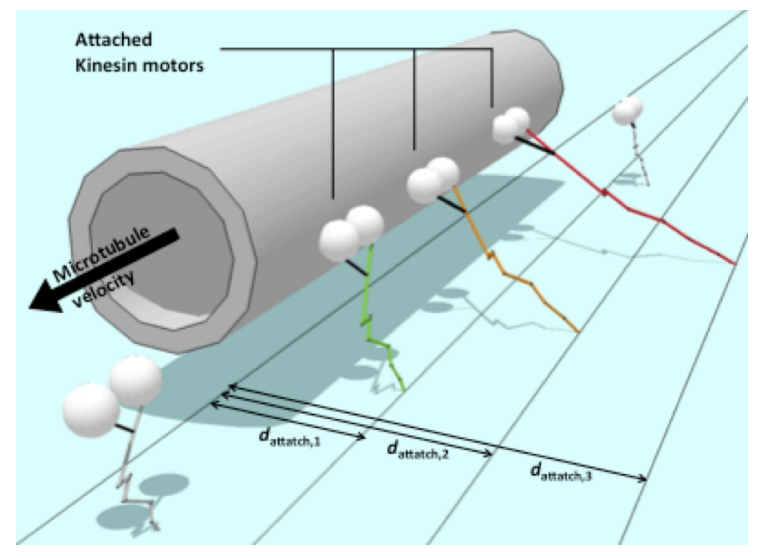

Figure 1. Motility assay schematic. Under our anharmonic tail stiffness hypothesis, the more kinesin motors attached further from the projection of the microtubule's axis on the surface (in red) are stiffer than the motors closer to the filament's axis (in green on the figure)

\section{Keywords}

Brownian Dynamics, Motility Assay, Motor Protein, Microtubule, Kinesin

\section{INTRODUCTION}

In a viscous medium, any velocity fluctuations observed during directed motion lead to energy dissipation and therefore to a loss of efficiency. These observed velocity fluctuations have previously been observed and quantified by a motional diffusion coefficient [3; 4]. A theoretical framework explaining these fluctuations as a consequence of the addition and removal of heterogeneous motors from the linear array of motors propelling the filament has been proposed by Sekimoto and Tawada [5]. Their model gave rise to a linear relationship between the motional diffusion coefficient and a factor given by the heterogeneity of motor effectiveness. We extended their model to motors with fixed steps and variable stiffnesses and obtained the following expression for the motional diffusion coefficient:

$$
D_{m}=\frac{\left\langle\left(k_{i}-\left\langle k_{i}\right\rangle\right)^{2}\right\rangle}{\left\langle k_{i}\right\rangle^{2}} \times \frac{v}{2 \rho}=\alpha_{k} \times \frac{v}{2 \rho}
$$

where $k_{i}$ is the stiffness of motor $i, v$ is the microtubule's velocity and $\rho$ the linear density of attached motors. We then used a

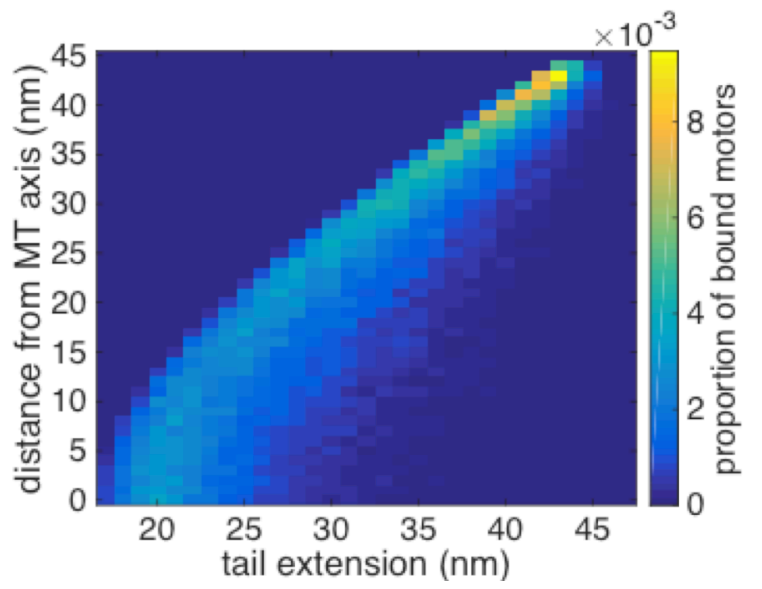

Figure 2. Brownian dynamics simulation results. Distribution of tail extension as a function of distance from the projection of the microtubule's axis on the surface 
Brownian dynamics simulation of the kinesin's head diffusion under an anharmonic potential to determine the stiffness distribution. This approach yields a theoretical value of 0.3 for the heterogeneity factor.

\section{RESULTS AND DISCUSSION 2.1 Results}

We use a Brownian Dynamics simulation of the kinesin head diffusion [1] to determine the distribution of kinesin tail extension. This consists in discretizing the following set of equations:

$$
\begin{gathered}
\frac{d x_{i}(t)}{d t}=\frac{1}{\zeta}\left(f_{i}^{k}+f^{r}\right) \\
\left\langle f^{r}\left(t_{1}\right) f^{r}\left(t_{2}\right)\right\rangle=2 k T \zeta \delta\left(t_{1}-t_{2}\right)
\end{gathered}
$$

where $f^{k}$ is the elastic force exerted on the diffusing head by the kinesin tail, $f^{r}$ is the random Langevin force, $\zeta$ is given by the Einstein relation for the diffusing tethered kinesin head $D^{\text {kinesin }}=$ $k T / \zeta$, and $D^{\text {kinesin }}=20 \mu \mathrm{m}^{2} / \mathrm{s}$ (from [2]).

We then use the freely jointed chain force-extension relationship to determine the stiffnesses from the extensions $r$ :

$$
r=\sum_{i=1}^{n} \operatorname{coth}\left(\frac{f^{k} l_{i}}{k T}\right)-\frac{k T}{f^{k} l_{i}}
$$

where $f^{k}$ is the force, $l_{i}$ is the Kuhn length of segment $i$. Under this approximation, the motors further from the microtubule tend to be stiffer (see Figure 1). We run $10^{4}$ trajectories for various kinesin attachment points on the surface to determine if the kinesin head binds to the microtubule, and when it binds, to determine the distribution of its extension $l_{i}$ (see Figure 2). We obtain a theoretical value of 0.3 for the heterogeneity constant $\alpha_{k}$.

\subsection{Discussion}

We can explicitly quantify the difference between the work done including fluctuations, $\langle\Delta W\rangle$ and the work done in the absence of fluctuations $\Delta \bar{W}$, against a viscous force $F$ :

$$
\begin{aligned}
& <\Delta W>-\Delta \bar{W}=\langle F \Delta x\rangle-\langle F\rangle\langle\Delta x\rangle \\
& <\Delta W>-\Delta \bar{W}=\zeta \frac{\left\langle\Delta x^{2}\right\rangle}{\Delta t}-\zeta \frac{\langle\Delta x\rangle^{2}}{\Delta t}
\end{aligned}
$$

where $\zeta$ is the viscosity of the medium. In our model, the filament displacement can be written as follows, since the filament is in equilibrium after each step:

$$
\Delta x=\frac{k_{i} d}{N<k_{i}>}
$$

We then obtain:

$$
<\Delta W>-\Delta \bar{W}=\alpha_{k} \Delta \bar{W}
$$

Therefore, the heterogeneity factor represents the proportion of extra work required to advance against a viscous force in the presence of fluctuations.

\section{CONCLUSION}

In this paper, we extended Sekimoto and Tawada's theoretical framework for the expression of motional diffusion coefficient to the kinesin motility assay with anharmonic kinesin tail stiffness, and showed that the heterogeneity coefficient is linked to variations in motor attachment geometry. We then used Brownian dynamics simulations to determine, for the first time, a theoretical value for this heterogeneity coefficient of 0.3 . Finally, we showed that this heterogeneity factor is the relative supplementary work needed for motion in the presence of fluctuations. These results can be applied to the engineering of more efficient motility assays by aligning motors. This increase in efficiency when motors are aligned can be generalized to all nano-devices driven by many heterogeneous motors.

\section{REFERENCES}

[1] Grassia, P.S., Hinch, E.J., and Nitsche, L.C., 1995. Computer-Simulations of Brownian-Motion of ComplexSystems. Journal of Fluid Mechanics 282(Jan 10), 373403. $\quad \mathrm{DOI}=\quad \mathrm{http} / / / \mathrm{dx}$.doi.org/Doi $10.1017 / \mathrm{S} 0022112095000176$.

[2] Hyeon, C. and Onuchic, J.N., 2007. Mechanical control of the directional stepping dynamics of the kinesin motor. Proceedings of the National Academy of Sciences of the United States of America 104, 44 (Oct 30), 17382-17387. $\mathrm{DOI}=\mathrm{http}: / / \mathrm{dx}$.doi.org/10.1073/pnas.0708828104.

[3] Imafuku, Y., Toyoshima, Y.Y., and Tawada, K., 1996. Fluctuation in the microtubule sliding movement driven by kinesin in vitro. Biophysical Journal 70, 2 (Feb), 878886. DOI $\quad$ http://dx.doi.org/10.1016/S00063495(96)79631-5.

[4] Nitta, T. and Hess, H., 2005. Dispersion in active transport by kinesin-powered molecular shuttles. Nano Letters 5, 7 (Jul), 1337-1342. DOI= http://dx.doi.org/10.1021/n1050586t.

[5] Sekimoto, K. and Tawada, K., 1995. Extended TimeCorrelation of in-Vitro Motility by Motor Protein. Physical Review Letters 75, 1 (Jul 3), 180-183. DOI= http://dx.doi.org/DOI 10.1103/PhysRevLett.75.180. 